\title{
Optimisation of reaction parameters for a novel polymeric additives as flow improvers of crude oil using response surface methodology
}

\author{
Ibrahim Elganidi $^{1} \cdot$ Basem Elarbe $^{1} \cdot$ Norida Ridzuan $^{1} \cdot$ Norhayati Abdullah $^{1}$
}

Received: 24 July 2021 / Accepted: 20 October 2021 / Published online: 29 October 2021

(c) The Author(s) 2021

\begin{abstract}
In recent years, polymeric additives have received considerable attention as a wax control approach to enhance the flowability of waxy crude oil. Furthermore, the satisfactory model for predicting maximum yield in free radical polymerisation has been challenging due to the complexity and rigours of classic kinetic models. This study investigated the influence of operating parameters on a novel synthesised polymer used as a wax deposition inhibitor in a crude oil pipeline. Response surface methodology (RSM) was used to develop a polynomial regression model and investigate the effect of reaction temperature, reaction time, and initiator concentration on the polymerisation yield of behenyl acrylate- $c o$-stearyl methacrylate- $c o$-maleic anhydride (BA-co-SMA-co-MA) polymer by using central composite design (CCD) approach. The modelled optimisation conditions were reaction time of $8.1 \mathrm{~h}$, reaction temperature of $102{ }^{\circ} \mathrm{C}$, and initiator concentration of $1.57 \mathrm{wt} \%$, with the corresponding yield of $93.75 \%$. The regression model analysis (ANOVA) detected an $R^{2}$ value of 0.9696 , indicating that the model can clarify $96.96 \%$ of the variation in data variation and does not clarify only $3 \%$ of the total differences. Three experimental validation runs were carried out using the optimal conditions, and the highest average yield is $93.20 \%$. An error of about $0.55 \%$ was observed compared with the expected value. Therefore, the proposed model is reliable and can predict yield response accurately. Furthermore, the regression model is highly significant, indicating a strong agreement between the expected and experimental values of BA-co-SMA-co-MA yield. Consequently, this study's findings can help provide a robust model for predicting maximum polymerisation yield to reduce the cost and processing time associated with the polymerisation process.
\end{abstract}

Keywords Waxy crude oil $\cdot$ Reaction parameters $\cdot$ Polymerisation yield $\cdot$ Flow improvers

\section{Introduction}

The possibility of wax deposition issues is one of the most significant difficulties in the processing and transporting of crude oil (Elarbe et al. 2021a). When the temperature of crude oil declines below the wax appearance temperature (WAT), the crystal wax starts to precipitate from the crude oil (Akinyemi et al. 2016). WAT is considered an essential indicator of wax deposition issues due to its conservative design criterion to prevent wax precipitation in production lines. Wax deposition increases the crude oil viscosity and flow line roughness and decreases the productive

Norhayati Abdullah

yatiabdullah@ump.edu.my

1 Faculty of Chemical \& Process Engineering Technology, Universiti Malaysia Pahang, 26300 Gambang, Pahang, Malaysia area, thereby increasing the pressure drop and decreasing the production rate. In extreme situations, wax deposition causes blockage of pipelines, as shown in Fig. 1, resulting in higher overhead costs or facility abandonment (Ridzuan and Al-Mahfadi 2017). To remediate the wax deposition issue, scholars have used various methods, such as thermal treatment, insertion of a chemical inhibitor, and mechanical treatment using pigging. If an appropriate approach is used in remediation, the associated cost will be drastically decreased. The most practical method to mitigate this problem is pre-treatment with polymeric additives, also known as pour point depressants (PPD) or flow improvers. In the pipeline industry, polymeric treatment with PPDs is widely utilized in small doses to decrease the crude oil pour point and gelation point, enhance the low-temperature flow properties and facilitate pipeline transportation (Jennings and Breitigam 2010; Aiyejina et al. 2011; Elbanna et al. 2017). 


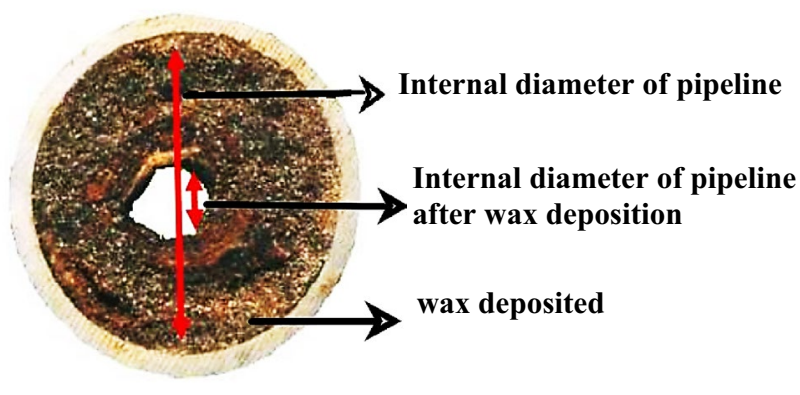

Fig.1 Plugged pipeline. Adapted from "A unified perspective on the phase behaviour of petroleum fluids" Source: (Mansoori 2009)

Many studies have been carried out to synthesise new forms of polymeric wax inhibitors to resolve wax deposition issues and increase the flow property of low-temperature waxy crude oil (Deka et al. 2020; Ahmed et al. 2021). Through experiments, previous studies and the current research aimed to improve synthesis methods of new products by decreasing the number of experiments and offering knowledge about the direct effects of additives on variables and interactions. This statistical technique has been successfully implemented in several fields (Deriase et al. 2012; El-Gendy et al. 2013a). Furthermore, empirical or semi-empirical models can predict responses under various experimental conditions without requiring assumptions. Therefore, the model's efficiency should be improved to increase yield without increasing costs. Applying the onefactor-at-a-time screening technique is a practical approach for determining the best processing conditions, specifically the range of each variable (Saha and Mazumdar 2019). However, this approach does not account for interactive effects among parameters and does not explain the full effect of the variables on the operation process. To avoid this obstacle, optimisation studies can be carried out using (RSM) (Aydar 2018).

RSM is a progressive critical technique for evolving pioneer methods, improving the model and formulation of new-found products, and maximising output (Mäkelä 2017). Optimization of the polymerization factors using RSM has several advantages over the classical optimization methods in which the one-variable-at-a-time method is used. Firstly, RSM offers a large amount of information from a small number of experiments. Indeed, classical techniques are time-consuming, and many experiments are needed to explain the behaviour of a system. Therefore, utilizing RSM will decrease the required amount of experimental runs for a quicker and more comprehensive investigation of the operating parameters and simultaneous interactions of parameters and modelling selected response variables (Nasouri et al. 2015, 2012). Secondly, RSM is a valuable instrument for considering multiple independent variables and their interactions that affect the objective process when various factors influence a polymerization yield (Song et al. 2014). Consequently, the interaction effect of the reaction parameters would be more critical such as synergism, antagonism. Furthermore, a polynomial equation describes the influence of associated variables in their corresponding coefficients, and this statistical model is a reference for optimisation research (Nuchitprasittichai and Cremaschi 2011). Subsequently, the model equation will easily clarify these effects for binary combinations of the independent parameters. Lastly, Central composite design (CCD) is a typical, efficient, and most widely employed RSM design (Ghelich et al. 2019). Therefore, CCD is ideal for delegating operational variables in various assessments by simplifying the number of design points and estimating an accurate curvature to provide pertinent details for testing lack-of-fit (Mateen et al. 2020).

On the other hand, the major disadvantage of RSM is to fit the data to a second-order polynomial. We could not say that the second-order polynomial well accommodates all systems containing curvature. Therefore, explaining the effect of reaction parameters with a second-order polynomial is not possible, especially when the curve is nonsymmetric. To overcome this, the data can be converted into another form explained by the second-order model, such as logarithmic transformations, and other linearization methods can be applied for this purpose (Baş and Boyacı 2007). In addition, if a second-order model hardly explains the system, one should choose a smaller range of independent parameters. It is possible to increase the accuracy of the model equation by working in a narrow range of the independent parameter. Still, it should be remembered that working in a limited range reduces the possibility of determination of the stationary point. Therefore, preliminary work becomes more critical for the decision of the independent parameter range (Wani et al. 2012). However, there was no nonsymmetric curvature data in the current study, and none of the transformation forms was required to apply to the model. Furthermore, the main limitation of this study is that this method requires screening of suitable ranges of the operating parameters, and the optimization result is restricted to specific extraction scales (Chan et al. 2017). In this section, a systematic way to determine the appropriate range of the operational parameters has been conducted in previous research by Elganidi, et al. (Elganidi et al. 2021).

RSM has been used in several studies in the polymer industry and related disciplines (Kaith et al. 2018; Ghumman et al. 2021; Kaur and Jindal 2019; Torğut et al. 2020; Davoudpour et al. 2015). Razali, et al. (2015) utilised RSM to investigate the grafting of poly diallyl dimethyl ammonium chloride to cassava starch with potassium persulphate as an initiator. The single and interactive effects of four variables, namely, initiator concentration, mole ratio, reaction 
time, and reaction temperature on grafting percentage, were analysed using CCD. The experimental yield under the optimum condition was similar to the value forecasted by their derived model, indicating the satisfactory performance of polymerisation. According to Aroonsingkarat and Hansupalak (2013), the four variables of processing conditions examined were reaction time, the number of a chain transfer agent, temperature, and percentage of deproteinised rubber. The research investigated the effect of the reaction parameters on monomer conversion in polystyrene and rubber graft copolymerisation by RSM via CCD. Elarbe et al. (2021b) studied the influence of processing variables on yield polymerisation by using CCD. The model is consistent and capable of appropriately forecasting yield response. The regression model has been proven to be extremely important and has a satisfactory outcome between the predicted and experimental stearyl acrylate-co-behenyl acrylate yields.

The current research's motivation is to synthesise a novel terpolymer and optimise polymerisation parameters, like initiator concentration, polymerisation time, and reaction temperature on the synthesised polymer' yield. This is because, to the best of our knowledge, no such work has been published to optimise the reaction parameters for the synthesis of poly(BA-co-SMA-co-MA) polymers using RSM. In previous research, Elganidi, et al. (2021) investigated the effect of four reaction parameters (temperature, time, initiator concentration, and mole ratio of monomer) on the yield of the free radical polymerisation of BA-coSMA-co-MA polymer by using OFAT method. However, this method cannot examine the variable interactions of the considered reaction (Elarbe et al. 2021a). Consequently, an experimental design approach was applied to evaluate the optimisation of the operational parameters of BA-co-SMAco-MA polymerisation. The statistical model was created to explain relationships among variables. As a result, the best possible response was explored under the desired condition and optimised process. In conclusion, the present research mainly aimed to analyse the influence of reaction parameters, including initiator concentration, reaction temperature, and time of reaction on polymerisation yield. Given that this statistical test and design can be used for process modelling and optimisation (Myers et al. 2016), CCD via RSM was used to plan experiments and create quadratic equation models for predicting the optimal conditions. Furthermore, these factors affecting investigational procedures were identified so that future researches could be designed to attain maximum yield of the polymerisation process.

The current study consists of the material and methodology used to synthesise the novel polymer and demonstrate how the experiments were designed and optimised using Design-Expert software. Secondly, the new regression model has been developed in the results and discussion section, and analysis of variance was applied to determine the significance value of this model. After that, verification of model and normality test using diagnostic plots were used to assess the goodness-of-fit of the proposed model. Then, the main critical factors that may influence the polymerisation process have been obtained by analysing the response surface. Lastly, before concluding with summary and conclusion, the experimental validation runs were conducted in triplicate to validate the predicted response variables.

\section{Materials and method}

\section{Materials}

This study used three main monomers for the synthesis process, including stearyl methacrylate, behenyl acrylate, and maleic anhydride. Also, toluene and benzoyl peroxide was used as a solvent and initiator, respectively. The chemical structure, molecular formula, purity, and suppliers of the used material were elaborated in Table 1.

\section{Polymerisation}

In a $250 \mathrm{~mL}$ three-necked glass flask, behenyl acrylate-costearyl methacrylate-co-maleic anhydride was polymerised utilising a free radical polymerisation method at a concentration of 1:1:1 mol ratios of monomers. A thermometer, a magnetic stirrer, and a nitrogen gas inlet (for the first $30 \mathrm{~min}$ ) for eliminating the existence of $\mathrm{O}_{2}$ in the reaction were fitted in the reactor. The monomer mixture was dissolved in $50 \mathrm{~mL}$ of toluene under continuous stirring at $400 \mathrm{rpm}$ for polymerisation at $90-110{ }^{\circ} \mathrm{C}$ for $7-9 \mathrm{~h}$. Benzoyl peroxide was employed in concentrations ranging from 1 to $2 \mathrm{wt} \%$ and dissolved in a proper quantity of toluene to initiate the reaction. The initiator solvent was applied to the reaction mixture drop by drop every $15 \mathrm{~min}$ for the first hour of the reaction. The mixture was allowed to cool at room temperature, washed three times with methanol, vacuum filtered, dried, and weighed to obtain terpolymer.

\section{Experimental design and optimisation}

The reaction parameters for the preparation of BA-coSAM-co-MA polymer were optimised. The experiment was conducted based on the central composite design of RSM. Design Expert 7.1.6 software was utilized to perform regression, design a model, explain experiments with multi-variable impacts, and minimise the number of experimental runs. This software has a wide range of designs, which consist of composite models, fractional factorials, and factorials, and can provide researchers with an assembly of numerical and mathematical RSM models. The experimental model was statistically analysed in terms of full quadratic, linear, and

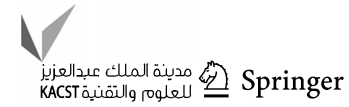


Table 1 Chemicals used in this study

\begin{tabular}{|c|c|c|c|c|c|}
\hline No & Type of chemicals & Structure & Molecular formula & Utilisation & Supplier \\
\hline 1 & $\begin{array}{l}\text { Behenyl acrylate (BA) } \\
(99 \%)\end{array}$ & & $\mathrm{C}_{25} \mathrm{H}_{48} \mathrm{O}_{2}$ & Monomer & Aladdin Chemicals \\
\hline 2 & $\begin{array}{l}\text { Stearyl methacrylate } \\
\text { (SMA) }(96 \%)\end{array}$ & & $\mathrm{C}_{21} \mathrm{H}_{40} \mathrm{O}_{2}$ & Monomer & \\
\hline 3 & $\begin{array}{l}\text { Maleic anhydride } \\
\text { (MA) }(<99 \%),\end{array}$ & & $\mathrm{C}_{4} \mathrm{H}_{2} \mathrm{O}_{3}$ & Monomer & \\
\hline 4 & $\begin{array}{l}\text { Benzoyl peroxide } \\
\text { (BPO) }(99 \%)\end{array}$ & & $\mathrm{C}_{14} \mathrm{H}_{10} \mathrm{O}_{4}$ & Initiator & Sigma- Aldrich \\
\hline 5 & Toluene $(\geq 99 \%)$ & & $\mathrm{C}_{6} \mathrm{H}_{5} \mathrm{CH}_{3}$ & Solvent & \\
\hline 6 & Methanol $(\geq 99 \%)$ & $-\mathrm{OH}$ & $\mathrm{CH}_{3} \mathrm{OH}$ & Precipitation & \\
\hline
\end{tabular}

interaction coefficients by ANOVA with F-test to determine the empirical correlation among the output and input variables. In addition, every model code was statistically evaluated, specifically the importance of $F$-values with $P \leq 0.05$, to improve the model. The $R^{2}$, adjusted $R^{2}$, expected $R^{2}$ values, adequate precision, and lack-of-fit of the models satisfied the recommended polynomial feature. The contour and response surface graphs were illustrated to visualise the input and output interactions.

The independent variables studied were reaction time $(A)$, reaction temperature $(B)$, and concentration of initiator $(C)$. Using CCD, each numeric factor fluctuated over five levels: plus and minus axial point (alpha), plus and minus 1 (factorial point), and centre point. The chosen centre points for each variable were reaction temperature of $100{ }^{\circ} \mathrm{C}$, reaction time of $8 \mathrm{~h}$, and initiator concentration of $1.5 \mathrm{wt} \%$; these values were selected because of the highest yield during polymerisation.
Table 2 demonstrates the experiment design levels and the range of independent variables used in this work. In addition, a three-factor five-level CCD was further studied. Twenty runs, including six replicate runs at the centre, six axial runs, and eight factorial runs, are needed based on a calculation using Eq. 1 (Owolabi et al. 2018).

$N=2^{n}+2 n+N c=2^{3}+2 * 3+6=20$

where $N$ is the number of experimental runs, and $n$ is the number of factors.

The model equation is determined, and model equation coefficients are expected. A full quadratic equation is a commonly used model in RSM. The $\alpha$-value was stable at 2 (face-cantered) for this model, and the response of the experimental model was obtained. The response (yield) can be employed to improve the experimental model in relation to the three parameters by using an additional-grade polynomial as follows in Eq. 2 (Bayuo et al. 2019). 
$Y=b_{o}+\sum_{i=1}^{n} b_{i} X_{i}+\sum_{i=1}^{n} b_{i i} X_{i i}+\sum_{i=1}^{n-1} \sum_{j=i+1}^{n} b_{i j} X_{i} X_{j}$

where $Y$ is the expected response, $b_{o}$ is the constant coefficient, $b_{i}$ is the linear coefficient, $b_{i i}$ is the quadratic equation, $b_{i j}$ is the interaction coefficient, and $X_{i}$ and $X_{j}$ are the coded values of the polymerisation factors. Table 2 demonstrates the order of runs, observed response (yield \%), and experimental design for the three independent variables and twenty experimental runs.

\section{Results and discussion}

OFAT experiments were conducted in our previous work (Elganidi et al. 2021). The optimal condition for the preparation of BA-co-SAM- $c o$-MA polymer comprised a mole ratio of $(1: 1: 1) \mathrm{wt} \%$ among the three monomers, reaction temperature of $100{ }^{\circ} \mathrm{C}$, initiator concentration of $1.5 \mathrm{wt} \%$ and reaction time of $8 \mathrm{~h}$, which were selected according to the highest yield under different conditions. In addition, CCD was employed to minimise the range of conditions with the maximum yield of BA-co-SAM-co-MA polymer and study the influence of variables on the yield.

\section{Development of regression model equation}

The reaction parameters were examined through CCD-based whereas the experimental runs along with the predicted and actual yields are shown in Table 3.

Analysis of variance (ANOVA) was applied to determine the significance value of the new model. The results show that the models are incredibly significant, with a significance level of $p<0.0001$; as such, the models can assist in predicting the response variable (yield). The created model's parameters $A, B, C, \mathrm{AB}, A^{2}, B^{2}$, and $C^{2}$, are significant $(p<0.0001)$. A second-order polynomial quadratic regression equation was defined in terms of coded factors in Eq. 3 and in terms of actual factors in Eq. 4.

$$
\begin{aligned}
\text { Yield }= & 93.35+0.75 A+1.21 B \\
& +0.59 C-0.58 A B+0.30 A C+0.46 B C \\
& -1.58 A^{2}-1.58 B^{2}-1.38 C^{2}
\end{aligned}
$$

The coded factor equation (Eq. 3) can be applied to expect the response for the provided level of each component. The high values of the coded variables are +1 , and the low ones are -1 . The coded equation is valuable for comparing factor coefficients to determine the relative influence of the variables. The equation in terms of a real variable (Eq. 4) can be utilised to estimate the response to such factor. The levels should be defined for each element in the original units. This equation ought not to be applied to verify the relative influence of each factor since the coefficients are calibrated to fit each factor's units and the intercept is not the centre of the model space (Anderson and Whitcomb 2017).

Yield $=-1132.41716+122.00943 *$ Time $+14.16315 *$ Temperature +12.95614

$*$ Concentration of Initiator $-0.23150 *$ Time $*$ Temperature $+2.39000 *$ Time

$*$ Concentration of Initiator $+0.36500 *$ Temperature $*$ Concentration of Initiator -6.30864

$*$ Time $^{2}-0.063086 *$ Temperature $^{2}-22.07455 *$ Concentration of Initiator $^{2}$.

RSM by utilising different ranges of three independent factors, namely, reaction time $(A)$, reaction temperature $(B)$, and initiator concentration $(C)$, to maximise the production yield of BA-co-SMA-co-MA polymer. The other reaction variables were maintained constant at their optimum levels obtained from the OFAT experiment. Twenty experimental runs were performed, each with a various combination of variables, according to CCD. The medium elements employed for RSM are presented in the process division,
The fitness of the model was justified by different parameters (Table 4). The current model evaluated the determination $\left(R^{2}\right)$ coefficient, adjusted $R^{2}$, predicted $R^{2}$, adequate precision, and 'lack-of-fit'. The $R^{2}$ value of 0.9696 reveals that the model could clarify $96.96 \%$ of variations in the data but does not clarify $3.04 \%$ of the overall differences. For an adequate model, the $R^{2}$ value should not be less than 0.75 (Myers et al. 2016).

\begin{tabular}{|c|c|c|c|c|c|c|c|}
\hline \multirow[t]{2}{*}{ Factor code } & \multirow[t]{2}{*}{ Factor name } & \multirow[t]{2}{*}{ Unit } & \multicolumn{5}{|c|}{ Factor levels for CCD } \\
\hline & & & $(-\alpha)$ & $(-1)$ & $(0)$ & $(+1)$ & $(+\alpha)$ \\
\hline$A$ & Reaction time & $\mathrm{h}$ & 7 & 7.5 & 8 & 8.5 & 9 \\
\hline$B$ & Reaction temperature & ${ }^{\circ} \mathrm{C}$ & 90 & 95 & 100 & 105 & 110 \\
\hline$C$ & Concentration of initiator & wt $\%$ & 1 & 1.25 & 1.5 & 1.75 & 2 \\
\hline
\end{tabular}

Table 2 Research factors and their coded levels 
Table 3 Experimental design matrix for the synthesis of BA-co-SAM-co-MA polymer

\begin{tabular}{|c|c|c|c|c|c|c|c|c|}
\hline \multirow[t]{2}{*}{ Run No } & \multicolumn{3}{|c|}{ Coded factor } & \multicolumn{3}{|c|}{ Actual Factor } & \multicolumn{2}{|l|}{$\%$ Conversion } \\
\hline & $A$ & $B$ & $C$ & $A(\mathrm{~h})$ & $B\left({ }^{\circ} \mathrm{C}\right)$ & $C(\mathrm{wt} \%)$ & $\begin{array}{l}\text { Observed } \\
\text { response (Yield } \\
\%)\end{array}$ & $\begin{array}{l}\text { Predicted } \\
\text { response (Yield } \\
\%)\end{array}$ \\
\hline 1 & -1 & -1 & -1 & 7.5 & 95 & 1.25 & 86.12 & 86.45 \\
\hline 2 & 1 & -1 & -1 & 8.5 & 95 & 1.25 & 87.85 & 88.51 \\
\hline 3 & -1 & 1 & -1 & 7.5 & 105 & 1.25 & 88.25 & 89.11 \\
\hline 4 & 1 & 1 & -1 & 8.5 & 105 & 1.25 & 89.45 & 88.86 \\
\hline 5 & -1 & -1 & 1 & 7.5 & 95 & 1.75 & 85.21 & 86.11 \\
\hline 6 & 1 & -1 & 1 & 8.5 & 95 & 1.75 & 89.92 & 89.37 \\
\hline 7 & -1 & 1 & 1 & 7.5 & 105 & 1.75 & 90.95 & 90.60 \\
\hline 8 & 1 & 1 & 1 & 8.5 & 105 & 1.75 & 91.56 & 91.54 \\
\hline 9 & -2 & 0 & 0 & 7.0 & 100 & 1.50 & 86.25 & 85.54 \\
\hline 10 & 2 & 0 & 0 & 9.0 & 100 & 1.50 & 88.15 & 88.55 \\
\hline 11 & 0 & -2 & 0 & 8.0 & 90 & 1.50 & 85.15 & 84.63 \\
\hline 12 & 0 & 2 & 0 & 8.0 & 110 & 1.50 & 89.25 & 89.46 \\
\hline 13 & 0 & 0 & -2 & 8.0 & 100 & 1.00 & 87.13 & 86.66 \\
\hline 14 & 0 & 0 & 2 & 8.0 & 100 & 2.00 & 88.85 & 89.01 \\
\hline 15 & 0 & 0 & 0 & 8.0 & 100 & 1.50 & 92.71 & 93.35 \\
\hline 16 & 0 & 0 & 0 & 8.0 & 100 & 1.50 & 93.82 & 93.35 \\
\hline 17 & 0 & 0 & 0 & 8.0 & 100 & 1.50 & 93.71 & 93.35 \\
\hline 18 & 0 & 0 & 0 & 8.0 & 100 & 1.50 & 93.84 & 93.35 \\
\hline 19 & 0 & 0 & 0 & 8.0 & 100 & 1.50 & 93.14 & 93.35 \\
\hline 20 & 0 & 0 & 0 & 8.0 & 100 & 1.50 & 93.21 & 93.35 \\
\hline
\end{tabular}

Saha and Mazumdar (Saha and Mazumdar 2019) argued that a high $R^{2}$ value does not necessarily mean a robust regression model. Such inference can only be made if the adjusted $R^{2}$ value is also high. The modified determination coefficient (adjusted $R^{2}=0.9422$ ) suggested that the generated model was highly significant, indicating consistent experimental and expected polymer yields. As a result, the model can accurately predict responses in a wide range of experimental variables. Rai et al. (2016) stated that the adjusted and predicted $R^{2}(0.8028)$ ought to be within $20 \%$ of each other to be in good agreement; this criterion is fulfilled in the present research. Therefore, the proposed model has $80.2 \%$ flexibility in predicting yield beyond the experimental variety of reaction circumstances. In the experiment, the adequate precision that computes the signal-to-noise ratio was 17.172 , indicating an adequate signal with less noise.

Table 5 indicates the ANOVA results for each term of the quadratic model. If the $F$-value is high and the $P$-value is less than 0.05 , then the term is significant. The linear terms $A, B$, and $C$, the quadratic terms $A 2$, $B 2$, and $C 2$, and the interaction term $\mathrm{AB}$ are significant (Table 4). The other variables do not affect the yield. Furthermore, a high $F$-value (35.40) with a low probability $(p=0.0001)$ indicates the high ability of the model to predict the results. The lack-of-fit of the model indicates
Table 4 Model fitness and coefficients in terms of coded factors

\begin{tabular}{lc}
\hline Factor & Coefficient estimate \\
\hline Intercept & 93.35 \\
$A$-Time & 0.7531 \\
$B$-Temperature & 1.21 \\
$C$-Initiator concentration & 0.5881 \\
$\mathrm{AB}$ & -0.5787 \\
$\mathrm{AC}$ & 0.2988 \\
$\mathrm{BC}$ & 0.4563 \\
$A^{2}$ & -1.58 \\
$B^{2}$ & -1.58 \\
$C^{2}$ & -1.38 \\
$R^{2}$ & 0.9696 \\
Adjusted $R^{2}$ & 0.9422 \\
Predicted $R^{2}$ & 0.8028 \\
Adeq precision & 17.1717 \\
\hline
\end{tabular}

the inconsistency between the expected and actual values on the pure error between replicates. According to the literature, the significance of 'lack-of-fit' can be attributed to the replicate measurements with the similarities of the repeating centre point data to each other (El-Gendy et al. $2013 b)$. The 'lack-of-fit $F$-value' of 3.93 implies that the 'lack-of-fit' is not significant relative to the pure error, 
and a $7.95 \%$ chance exists that the high $F$-value occurs due to noise. This relatively low probability $(<10 \%)$ does not trouble the model fitting (Usman et al. 2019). Nonsignificant 'lack-of-fit' can be used to fit the model to estimate the expected result accurately.

\section{Validation and verification of model and normality test}

Diagnostic plots, such as residuals normal probability, expected against actual values, residual versus predicted values, and standardised residuals against run plot, were used to determine the goodness-of-fit of the proposed model. In a normal probability plot, the residuals obey a straight line to the normal probability distribution (Cavazzuti 2012). Furthermore, studentised residuals are assumed to be more efficient than standardised residuals in detecting outlying responses (Fox 2015). Figure 2a demonstrates the normality plot of internally studentised residuals. The highest number of colour points representing the polymerisation yield is located in a tight range on a regular probability line, and the minor-significant points deviate from the normal line. The residual's independence and normality as well as its high validity for approximating the established quadratic regression model were confirmed by a satisfactory normal distribution with a random deviation of model predictions from the actual results (El-Gendy et al. 2013b; Kleijnen 2015). The predicted and actual yields were plotted as a parity plot of the regression model to identify values that the model cannot easily predict. Figure $2 b$ shows the plot of the polymerisation yield, indicating a relatively regular random scatter of spots assembled at the diagonal axis. This graph is relatively linear, passing through the origin, indicating that the observed (experimentally) polymerisation yields with a marginal variance are in good agreement with the expected values determined using optimisation methodology (Saeed et al. 2015). Consequently, the plot of residuals versus ascending expected responses measures the constant variance principle and shows a random scatter (steady range of residuals across the graph). The quadratic model is suitable and appropriate for the experimental results, and no further transformation of the data series is necessary. Figure $2 c$ characterises residuals against the experimental run order, which allows checking for lurking variables that may have influenced the response. The plot signifying a random regular scatter of points and lack an obvious pattern and infrequent structure indicates the fitness of the model. The plot of internally studentised residuals versus the expected values in Fig. $2 d$ shows that all colour points corresponding to yield are randomly scattered and situated within limits near to zero-axis in the range between \pm 2.0 . The expected values are very similar to the actual values; as such, the model effectively captures the correlation between yield and process conditions (Ghelich et al. 2019). Therefore, the model is adequate, and no independent or constant variance assumption violation exists in all runs. The model can be effectively employed to navigate the design space.

\section{Analysis of response surface}

RSM is used for modelling and analysis to optimise a related process response (output variable) dependent on many experimental parameters and create a multivariate mathematical estimation model. In multivariate optimisation, the relationship of various independent variables to the process should be assessed (Mukherjee et al. 2019). Figures 3, 4, and 5 show the three-dimensional quadratic response surfaces and the two-dimensional contour plots for different independent variables such as reaction time, reaction temperature, and initiator concentration concerning polymerisation
Table 5 Analysis of variance (ANOVA) for response surface quadratic model

\begin{tabular}{lcrrrrl}
\hline Source & Sum of Squares & Df & Mean Square & $F$-Value & $P$-value & \\
\hline Model & 164.41 & 9 & 18.27 & 35.40 & $<0.0001$ & significant \\
$A$-Reaction Time & 9.08 & 1 & 9.08 & 17.59 & 0.0018 & \\
$B$-Reaction Temp & 23.30 & 1 & 23.30 & 45.16 & $<0.0001$ & \\
$C$-Initiator Conc & 5.53 & 1 & 5.53 & 10.72 & 0.0084 & \\
AB & 2.68 & 1 & 2.68 & 5.19 & 0.0459 & \\
AC & 0.71 & 1 & 0.71 & 1.38 & 0.2667 & \\
BC & 1.67 & 1 & 1.67 & 3.23 & 0.1026 & \\
$A^{2}$ & 62.54 & 1 & 62.54 & 121.20 & $<0.0001$ & \\
$B^{2}$ & 62.54 & 1 & 62.54 & 121.20 & $<0.0001$ & \\
$C^{2}$ & 47.86 & & 47.86 & 92.74 & $<0.0001$ & \\
Residual & 5.16 & 10 & 0.52 & & & \\
Lack-of-fit & 4.11 & 5 & 0.82 & 3.93 & & not significant \\
Pure error & 1.05 & 5 & 0.21 & & & \\
\hline
\end{tabular}


Fig. 2 Diagnostic plots for polymerisation yield: a normal distribution, $\mathbf{b}$ predicted versus actual values, c studentised residuals versus run number and d studentised residuals versus predicted values
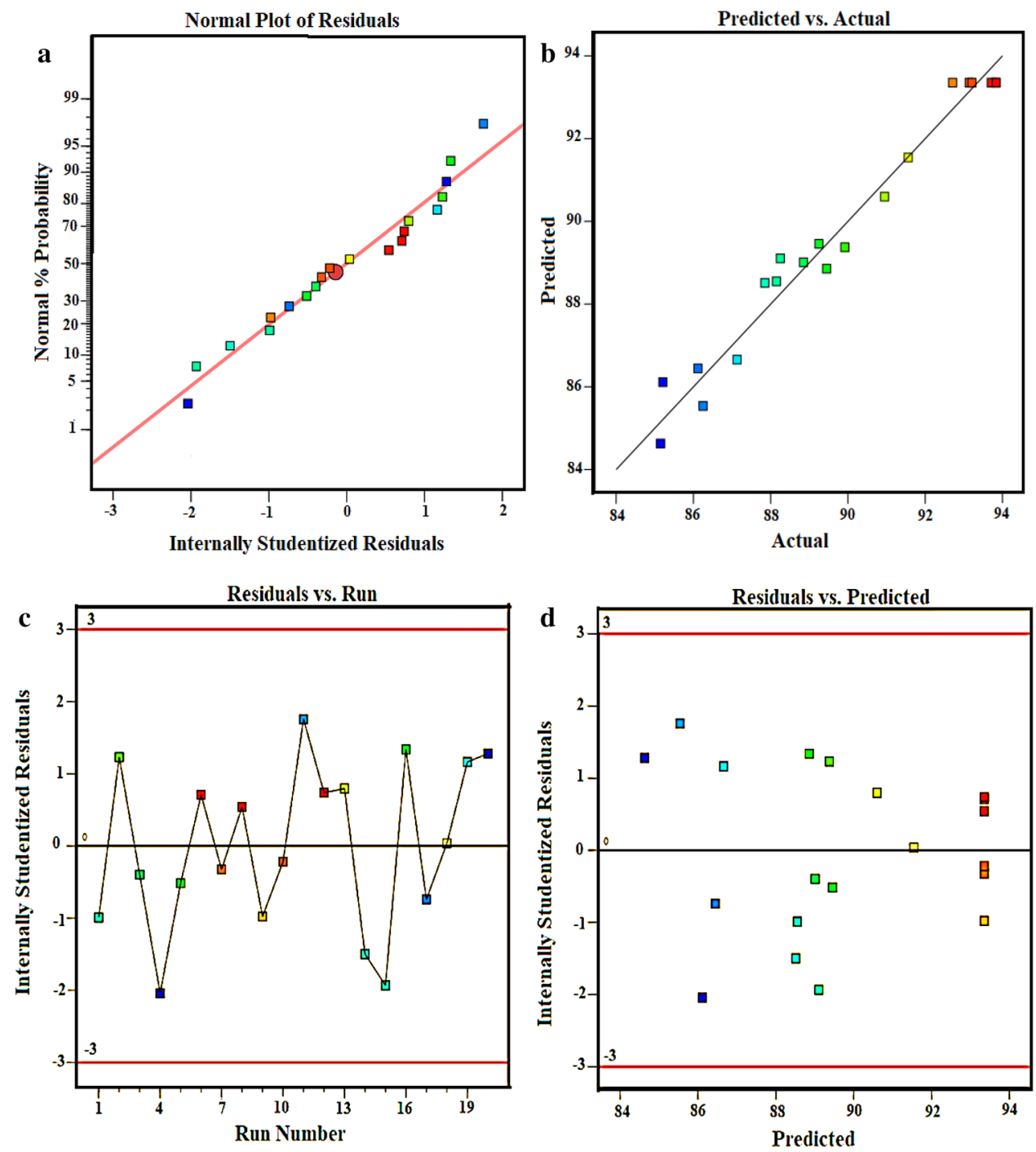

efficiency. Each figure shows the effect of changing two variables on the polymerisation yield while maintaining the third variable at zero levels. The plots reveal the correlated fitted response surface for the optimal design of reaction parameters to maximise the polymer yield (\%) and depict corresponding surface plots using dominant variables. Furthermore, the nature of the curvature of the plots illustrates the intensity of the interaction of the process variables (Karri et al. 2018).

The geometry of the contour plot shows the combined influence of the independent variables on the response parameter. All reaction surface plots and the resulting contour map have a design stage (Figs. 3a-c. The highest yield percentage is located within the design boundaries (Mukherjee et al. 2020). The structure of the contour map is crucial to predict whether the combined interactions between variables are relevant (Mukherjee et al. 2019). Moreover, the geometry of the contour map shows the actuality and degree of interactions between independent variables. If the contours are circular, then the combined interfaces of the operating variables might be less prominent; if the contour lines are elliptical, then the accumulative mutual combination of the procedure variables is more prominent (Tanyildizi et al. 2005). Elliptical contours are acquired when absolute shared interactions exist between operational variables. The lowest possible surface bound shows the highest yield percentage in the contour diagram. As reported by Myers et al. (2016), if the graphical displays could be easily constructed (Fig. 3a-c), then the optimisation process would be straightforward.

A response surface may occur when approximating a yield response, which we can assume to be operating near the surface's maximum points. Therefore, the inspection of the contour plots indicates that the yield is maximised under the condition of reaction of $8.1 \mathrm{~h}$, temperature of $102{ }^{\circ} \mathrm{C}$, and initiator concentration of $1.57 \mathrm{wt} \%$. The defined optimal 
Fig. 3 a Contour plot of yield versus reaction temperature and initiator concentration b Contour plot of yield versus reaction time and initiator concentration c Contour plot of yield versus A-reaction time and B-reaction temperature

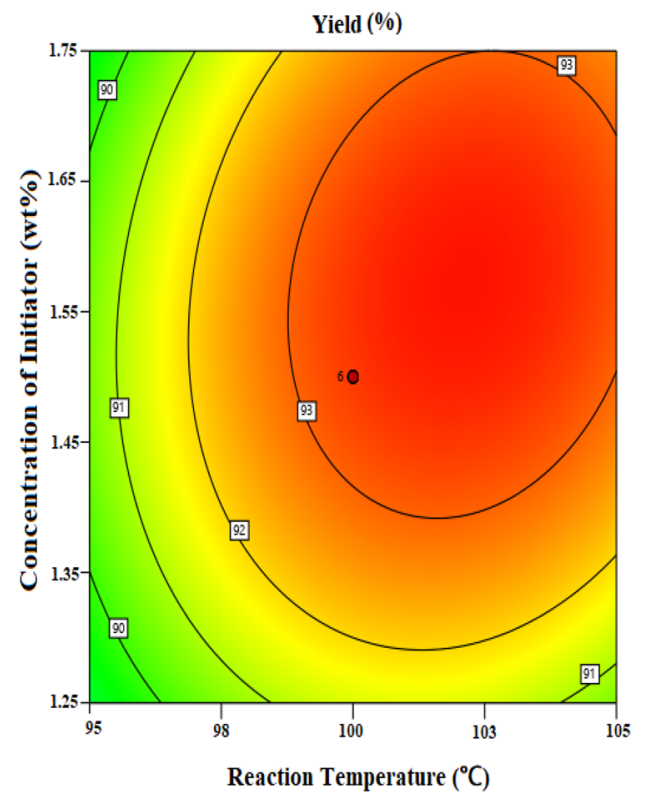

(a) Contour plot of yield versus reaction temperature and initiator concentration

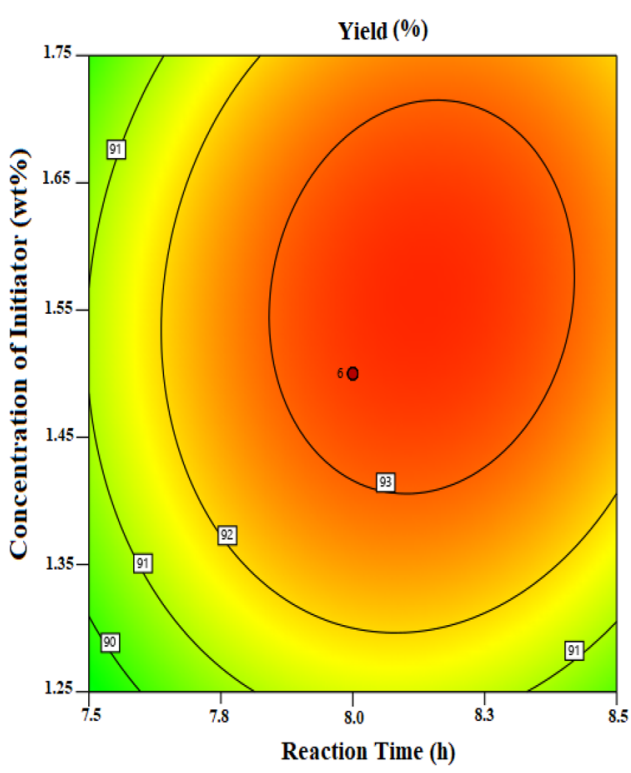

(b) Contour plot of yield versus reaction time and initiator concentration Yield (\%)

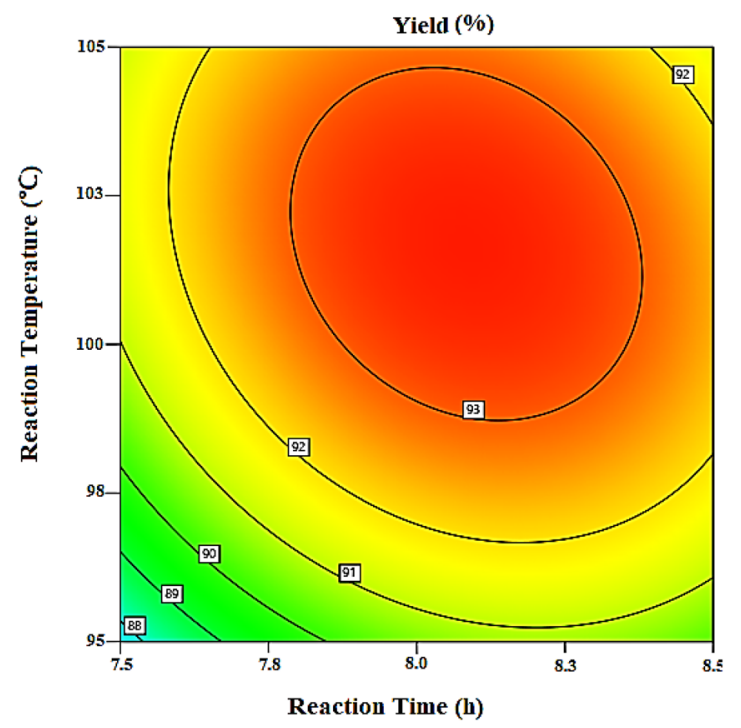

(c) Contour plot of yield versus (A) reaction time and (B) reaction temperature conditions were confirmed by repeating the experiments to eliminate errors. The findings were reliable and statistically significant within the range of the experimental parameters chosen.

Figure 4 shows the interactive effect of initiator concentration ranging from 1.25 to $1.75 \mathrm{wt} \%$ and reaction temperature of $95-105^{\circ} \mathrm{C}$ on the yield of polymerisation at a constant reaction duration of $8 \mathrm{~h}$. The polymerisation yield increased when the reaction temperature was increased to $100{ }^{\circ} \mathrm{C}$ with increasing benzoyl peroxide concentration to $1.50 \mathrm{wt} \%$ and reached the maximum under this condition parameter. With increasing temperature, the polymerisation yield increased uneventfully in a given time due to an increased decomposition rate of the initiator. The number of free radicals and their mobility also increased, resulting in a higher yield. Furthermore, the increase in the reaction temperature decreased the viscosity, thereby enhancing the monomer movement. As such, the used monomers (BA, SMA, and MA) easily spread to the vicinity of the synthesised polymer backbone, promoting the polymerisation reaction and increasing polymerisation yield, similar to the report of Wang et al. (2020). As the reaction temperature and initiator concentration were raised to a certain degree, the homopolymerisation, chain transition, and termination reactions were accelerated, resulting in a decrease in yield and a lowering of the curves; thus, the optimum reaction 


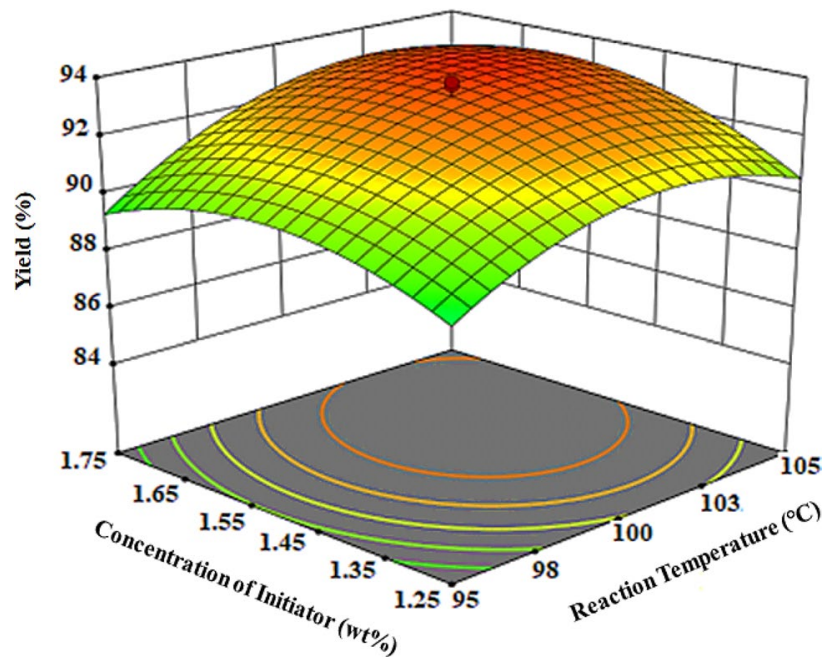

Fig. 4 Model interaction plot between initiator concentration and temperature on yield

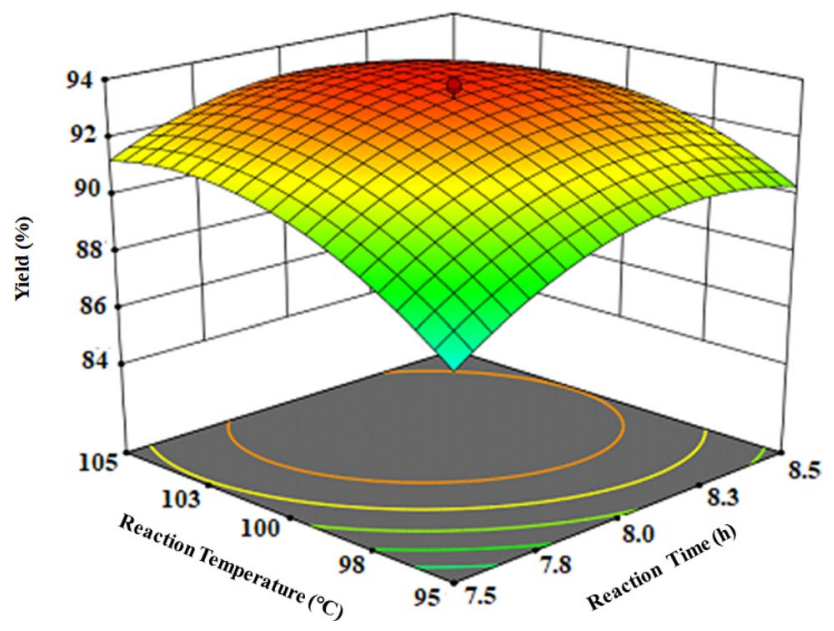

Fig. 5 Model interaction plot between temperature and time on yield

parameters should be a reaction temperature of $100{ }^{\circ} \mathrm{C}$ and BPO concentration of $1.5 \mathrm{wt} \%$.

The optimum yield was $92.71 \%$, which was obtained using the reaction temperature of $100{ }^{\circ} \mathrm{C}$ and the initiator concentration of $1.50 \mathrm{wt} \%$. Thus, the two process variables have a net positive interactive impact, indicating yield sensitivity to temperature and initiator concentration.

Figure 5 shows the interactive influence of the reaction temperature of $95-105^{\circ} \mathrm{C}$ and reaction time of $7.5-8.5 \mathrm{~h}$ on the yield of polymerisation at a constant initiator concentration of $1.50 \mathrm{wt} \%$. The yield increased with the rapid increase in the reaction temperature compared with prolonged reaction time. The increment in the temperature from 95 to $100{ }^{\circ} \mathrm{C}$ and the time from 7.5 to $8 \mathrm{~h}$ increased the polymerisation yield to the optimum level. The augmentation in temperature with time enhanced the polymerisation of monomers, the propagation reaction, and the chain transfer reaction (Arslan et al. 2021). Increasing the temperature improved the yield by elevating the concentration of radicals in the medium. The mobility of the monomer molecules accelerated the diffusion of the monomers into the polymer backbone. As the reaction time increased, the polymerisation yield increased and reached the optimum level after $8 \mathrm{~h}$ of reaction; no increase was observed in the yield after this time. The yield increased due to the increase in chain growth that joined on the polymer backbone and the formation of new chains onto the synthesised polymer.

The highest polymerisation yield was obtained at the glass transition temperature and above because the molecules obtained mobility and allowed for the diffusion of new molecular forms to enter the polymerisation process. The slight decrease in the polymerisation yield when the temperature is above $100{ }^{\circ} \mathrm{C}$ and after $8 \mathrm{~h}$ of reaction can be associated with the more dominant reactions of termination with increasing temperature and the termination of the initiator radicals by binding among themselves (Wang et al. 2020; Temoçin and Yiğitoğlu 2009). In conclusion, a significant positive interaction exists between the reaction temperature and reaction time. Increasing the reaction temperature positively affects the polymerisation yield because more reactive species have sufficient energy to overcome the barrier corresponding to the activation energy, resulting in faster and more complete reaction; this result is similar to those obtained by dos Santos et al. (2016). The highest yield reached $93.84 \%$ under the optimal condition of $8 \mathrm{~h}$ and $100{ }^{\circ} \mathrm{C}$. The yield increased with increasing reaction time as well as reaction temperature at the optimum initiator concentration of $1.5 \mathrm{wt} \%$.

Figure 6 shows the interactive influence of reaction time and initiator concentration on polymerisation yield. The reaction time was varied from 7.5 to $8.5 \mathrm{~h}$, and the concentration of benzoyl peroxide was between 1.25 and $1.75 \mathrm{wt} \%$, while the reaction temperature was maintained at $100{ }^{\circ} \mathrm{C}$. The yield increased with increasing initiator concentration compared with that of the reaction temperature. Furthermore, the polymerisation yield increased with increasing reaction time of up to $8 \mathrm{~h}$ due to an increase in the active sites on the synthesised polymer backbone. Above $8 \mathrm{~h}$, the polymerisation yield slightly decreased and then began to level off and reached a plateau because of the monomer and initiator concentration depletion, leading to a lack of polymerising sites on the polymer backbone. The yield increased to $92.71 \%$ when increasing 
the concentration of BPO to $1.50 \mathrm{wt} \%$ after $8 \mathrm{~h}$ of reaction and then decreased with a further increase in the BPO concentration and reaction time. This phenomenon commonly occurs and has been reported by various authors (Elella et al. 2018; Badwaik et al. 2016). The free radical concentration of the BPO molecules in the polymerisation medium increases with increasing BPO concentration with time. The radicals abstract a hydrogen atom from the monomer macromolecules and form active sites on the polymer backbone. Increasing the BPO concentration increases the number of radical species. These radical chains activate the polymeric chains to undergo a chain transfer reaction with the monomer macromolecules, leading to the formation of a high number of active sites on the polymer backbone. Therefore, the yield increases with the chain transfer and hydrogen abstraction reactions.

However, the increase in the BPO concentration and reaction time above the critical values of $1.5 \mathrm{wt} \%$ and $8 \mathrm{~h}$ led to excessive radicals in the polymerisation medium. Thus, the rate of termination reaction increased while the polymerisation yield decreased (Makhlouf et al. 2007; Işıklan et al. 2010). In conclusion, a positive interaction exists between the reaction time and concentration of the initiator, and the highest yield was $92.71 \%$. The yield continues to increase with increasing reaction time and initiator concentration until reaching the critical point, at which the yield did not improve anymore.

In summary, the highest yield was found to be $93.84 \%$, and the critical factors that influenced polymerisation are reaction time and temperature.

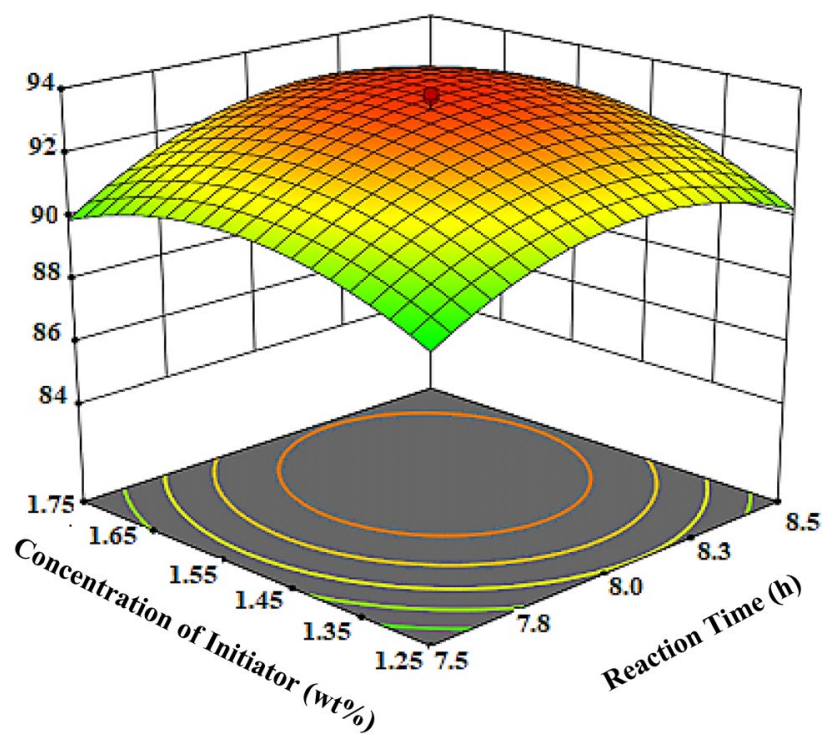

Fig. 6 Model interaction plot between initiator concentration and time on yield

\section{Optimisation and validation}

The optimisation process was carried out to determine the optimum value of the reaction parameters on the polymerisation yield by using Design Expert 6.0.7 software. The desired goal for each operational condition (reaction time, reaction temperature, and initiator concentration) was selected within the studied range. The yield was defined as the maximum in the software system to obtain the best performance. The software program integrates individual desirability into a single number and then optimises the function based on the response goal (Antony 2014).

. After applying the desirability function, the optimum response (yield) was $93.75 \%$ at reaction time of $8.1 \mathrm{~h}$, reaction temperature of $102{ }^{\circ} \mathrm{C}$, and initiator concentration of $1.57 \mathrm{wt} \%$. Under the optimum condition, experimental validation runs were conducted in triplicate, and the average yield obtained was $93.20 \%$ (Table 6).

The residual between the predicted responses was $93.75 \%$, and the experimental response was $93.20 \%$. Therefore, an error of only 0.55 existed, indicating that the quadratic regression model was valid and accurate in predicting the response ( $Y$ ) (Garba et al. 2016). Hence, the models and the optimum operating conditions developed for the factors were valid and applicable in predicting response variables.

\section{Summary and conclusions}

- RSM based on CCD enhances the experimental model building and can be applied to evaluate, predict and optimise process factors.

- The single and combined effects of three reaction parameters (reaction temperature, reaction time, and initiator concentration) on the yield of BA-co-SMA-co-MA in radical solution polymerisation were investigated.

- The optimum conditions were reaction time of $8.1 \mathrm{~h}$, reaction temperature of $102{ }^{\circ} \mathrm{C}$, initiator concentration of $1.57 \mathrm{wt} \%$, and the optimised yield was $93.20 \%$.

- The ANOVA results of the regression model revealed an $\mathrm{R}^{2}$ value, indicating that the model could explain $96.96 \%$ of data heterogeneity, and only $3 \%$ of the total variations were not explained by the model.

- Under the optimum condition, experimental validation runs were conducted in triplicate, and the maximum average yield was obtained.

- The model is extremely significant, confirming the close agreement between the experimental and predicted values of BA-co-SMA-co-MA yield because of the neglected error between the predicted and actual values. 
Table 6 Model validation

\begin{tabular}{|c|c|c|c|c|c|c|}
\hline \multirow[t]{2}{*}{ Model desirability } & \multirow[t]{2}{*}{ Reaction time (h) } & \multirow[t]{2}{*}{ Reaction temperature $\left({ }^{\circ} \mathrm{C}\right)$} & \multirow[t]{2}{*}{ Concentration of initiator (wt $\%$ ) } & \multicolumn{3}{|l|}{ Yield (\%) } \\
\hline & & & & Experimental & Predicted & Error \\
\hline 0.990 & 8.1 & 102 & 1.57 & 93.20 & 93.75 & 0.55 \\
\hline
\end{tabular}

Acknowledgements The authors would like to thank the Faculty of Chemical and Process Engineering Technology at University Malaysia Pahang for funding this research through PGRS 1903103 and RDU1803172 scheme

Funding This research was funded by University Malaysia Pahang through PGRS 1903103 and RDU1803172 scheme.

\section{Declaration}

The authors declare that they have no known competing financial interests or personal relationships that could have influenced the work reported in this paper.

Open Access This article is licensed under a Creative Commons Attribution 4.0 International License, which permits use, sharing, adaptation, distribution and reproduction in any medium or format, as long as you give appropriate credit to the original author(s) and the source, provide a link to the Creative Commons licence, and indicate if changes were made. The images or other third party material in this article are included in the article's Creative Commons licence, unless indicated otherwise in a credit line to the material. If material is not included in the article's Creative Commons licence and your intended use is not permitted by statutory regulation or exceeds the permitted use, you will need to obtain permission directly from the copyright holder. To view a copy of this licence, visit http://creativecommons.org/licenses/by/4.0/.

\section{References}

Ahmed SM, Khidr TT, Ali ES (2021) Preparation and evaluation of polymeric additives based on poly (styrene-co-acrylic acid) as pour point depressant for crude oil. J Dispers Sci Technol 20(2):1-8

Aiyejina A, Chakrabarti DP, Pilgrim A, Sastry M (2011) Wax formation in oil pipelines: a critical review. Int J Multiph Flow 37(7):671-694

Akinyemi OP, Udonne JD, Efeovbokhan VE, Ayoola AA (2016) A study on the use of plant seed oils, triethanolamine and xylene as flow improvers of Nigerian waxy crude oil. J Appl Res 14(3):195-205

Anderson MJ, Whitcomb PJ (2017) DOE simplified: practical tools for effective experimentation, 3rd edn. CRC Press, United States

Antony J (2014) Design of experiments for engineers and scientists, 2nd edn. Elsevier, Scotland, UK

Aroonsingkarat K, Hansupalak N (2013) Prediction of styrene conversion of polystyrene/natural rubber graft copolymerization using reaction conditions: central composite design versus artificial neural networks. J Appl Polym Sci 128(4):2283-2290

Arslan M, Günay K, Gök ZG, Yiğitoğlu M (2021) Synthesis and characterization of poly (ethylene terephthalate) fibers grafted with $\mathrm{N}$-(hydroxymethyl) acrylamide by free radical: its application in elimination of Congo red. Polym Bull 78(3):1535-1550

Aydar AY (2018) Utilization of response surface methodology in optimization of extraction of plant materials. In: Statistical approaches with emphasis on design of experiments applied to chemical processes, pp 157-169. https://doi.org/10.5772/intechopen.73690

Badwaik HR, Sakure K, Alexander A, Dhongade H, Tripathi DK (2016) Synthesis and characterisation of poly (acryalamide) grafted carboxymethyl xanthan gum copolymer. Int J Biol Macromol 85(1):361-369

Baş D, Boyacı IH (2007) Modeling and optimization I: usability of response surface methodology. J Food Eng 78(3):836-845

Bayuo J, Pelig-Ba KB, Abukari MA (2019) Optimization of adsorption parameters for effective removal of lead (II) from aqueous solution. Phys Chem Indian J 14:1-25

Cavazzuti M (2012) Optimization methods: from theory to design scientific and technological aspects in mechanics. Springer, Berlin, Germany

Chan C-H, Yusoff R, Ngoh GC (2017) An energy-based approach to scale up microwave-assisted extraction of plant bioactives. In: Grumezescu AM, Holban AM (eds) Ingredients extraction by physicochemical methods in food. Academic Press, pp 561-597. https://doi.org/10.1016/B978-0-12-811521-3.00015-6

Davoudpour Y et al (2015) Optimization of high pressure homogenization parameters for the isolation of cellulosic nanofibers using response surface methodology. Ind Crops Prod 74(1):381-387

Deka B, Sharma R, Mahto V (2020) Synthesis and performance evaluation of poly (fatty esters-co-succinic anhydride) as pour point depressants for waxy crude oils. J Petrol Sci Eng 191(1):107153

Deriase S, El-Gendy NS, Nassar H (2012) Enhancing biodegradation of dibenzothiophene by Bacillus sphaericus HN1 using factorial design and response surface optimization of medium components. Energy Sour Part A Recover Util Environ Effects 34(22):2073-2083

Elarbe B, Elganidi I, Ridzuan N, Abdullah N, Yusoh K (2021a) Influence of poly (stearyl acrylate co-behenyl acrylate) as flow improvers on the viscosity reduction of Malaysian crude oil. Mater Today Proc 42(1):201-210

Elarbe B, Elganidi I, Abdullah N, Yusoh K, Ridzuan N (2021b) Optimization and modeling of reactive conditions for free radical solution polymerization of SA-co-BA copolymer based on the yield using response surface methodology. Malays J Fundam Appl Sci 17(1):50-55

Elbanna S, Abd El Rhman A, Al-Hussaini A, Khalil S (2017) Synthesis and characterization of polymeric additives based on $\alpha$-Olefin as pour point depressant for Egyptian waxy crude oil. Pet Sci Technol 35(10): 1047-1054

Elella MHA, Mohamed RR, Sabaa MW (2018) Synthesis of novel grafted hyaluronic acid with antitumor activity. Carbohyd Polym 189:107-114

Elganidi I, Elarbe B, Abdullah N, Ridzuan N (2021) Synthesis of a novel terpolymer of (BA-co-SMA-co-MA) as pour point depressants to improve the flowability of the Malaysian crude oil. Mater Today Proc 42(1):28-32

El-Gendy NS, Madian HR, Amr SSA (2013a) Design and optimization of a process for sugarcane molasses fermentation by Saccharomyces cerevisiae using response surface methodology. Int J Microbiol 2013(1):9-15

El-Gendy NS, Madian HR, Amr SSA (2013) Design and optimization of a process for sugarcane molasses fermentation by 
Saccharomyces cerevisiae using response surface methodology. Int J Microbiol. https://doi.org/10.1155/2013/815631

Fox J (2015) Applied regression analysis and generalized linear models, 3rd edn. Sage Publications, United states of America

Garba ZN, Bello I, Galadima A, Lawal AY (2016) Optimization of adsorption conditions using central composite design for the removal of copper (II) and lead (II) by defatted papaya seed. Karbala Int J Modern Sci 2(1):20-28

Ghelich R, Jahannama MR, Abdizadeh H, Torknik FS, Vaezi MR (2019) Central composite design (CCD)-Response surface methodology (RSM) of effective electrospinning parameters on PVPB-Hf hybrid nanofibrous composites for synthesis of HfB2-based composite nanofibers. Compos B Eng 166:527-541

Ghumman ASM, Shamsuddin R, Nasef MM, Yahya WZN, Abbasi A (2021) Optimization of synthesis of inverse vulcanized copolymers from rubber seed oil using response surface methodology. Polymer 219:123553

Işıklan N, Kurşun F, İnal M (2010) Graft copolymerization of itaconic acid onto sodium alginate using benzoyl peroxide. Carbohyd Polym 79(3):665-672

Jennings DW, Breitigam J (2010) Paraffin inhibitor formulations for different application environments: From heated injection in the desert to extreme cold arctic temperatures. Energy Fuels 24(4):2337-2349

Kaith BS, Shanker U, Gupta B, Bhatia JK (2018) RSM-CCD optimized In-air synthesis of photocatalytic nanocomposite: Application in removal-degradation of toxic brilliant blue. React Funct Polym 131:107-122

Karri RR, Tanzifi M, Yaraki MT, Sahu J (2018) Optimization and modeling of methyl orange adsorption onto polyaniline nano-adsorbent through response surface methodology and differential evolution embedded neural network. J Environ Manage 223:517-529

Kaur K, Jindal R (2019) Exploring RSM-CCD-optimized chitosan-/ gelatin-based hybrid polymer network containing $C P M-\beta-C D$ inclusion complexes as controlled drug delivery systems. Polym Bull 76(7):3569-3592

Kleijnen JPC (2015) Response surface methodology. In: Fu M (ed) Handbook of simulation optimization. International series in operations research \& management science, vol 216. Springer, New York, NY. https://doi.org/10.1007/978-1-4939-1384-8_4

Mäkelä M (2017) Experimental design and response surface methodology in energy applications: a tutorial review. Energy Convers Manage 151:630-640

Makhlouf C, Marais S, Roudesli S (2007) Graft copolymerization of acrylic acid onto polyamide fibers. Appl Surf Sci 253(12):5521-5528

Mansoori GA (2009) A unified perspective on the phase behaviour of petroleum fluids. Int J Oil Gas Coal Technol 2(2):141-167

Mateen QS, Khan SU, Islam DT, Khan NA, Farooqi IH (2020) Copper (II) removal in a column reactor using electrocoagulation: Parametric optimization by response surface methodology using central composite design. Water Environ Res 92(9):1350-1362

Mukherjee A, Datta D, Halder G (2019) Synthesis and characterisation of rice-straw-based grafted polymer composite by free radical copolymerisation. Indian Chem Eng 61(2):105-119

Mukherjee A, Karmakar B, Halder G (2020) Response surface optimized free radical grafting of methyl methacrylate on de-lignified rice straw for evaluating its application potential as flame retardant roofing material. Chem Eng J Adv 1:100007

Myers RH, Montgomery DC, Anderson-Cook CM (2016) Response surface methodology: process and product optimization using designed experiments, 4th edn. Wiley, Canada

Nasouri K, Shoushtari AM, Mojtahedi MRM (2015) Effects of polymer/solvent systems on electrospun polyvinylpyrrolidone nanofiber morphology and diameter. Polym Sci, Ser A 57(6):747-755

Nasouri K, Bahrambeygi H, Rabbi A, Shoushtari AM, Kaflou A (2012) Modeling and optimization of electrospun PAN nanofiber diameter using response surface methodology and artificial neural networks. J Appl Polym Sci 126(1):127-135

Nuchitprasittichai A, Cremaschi S (2011) Optimization of CO2 capture process with aqueous amines using response surface methodology. Comput Chem Eng 35(8):1521-1531

Owolabi RU, Usman MA, Kehinde AJ (2018) Modelling and optimization of process variables for the solution polymerization of styrene using response surface methodology. J King Saud Univ Eng Sci 30(1):22-30

Rai A, Mohanty B, Bhargava R (2016) Supercritical extraction of sunflower oil: a central composite design for extraction variables. Food Chem 192:647-659

Razali M, Ismail H, Ariffin A (2015) Graft copolymerization of polyDADMAC to cassava starch: evaluation of process variables via central composite design. Ind Crops Prod 65:535-545

Ridzuan N, Al-Mahfadi M (2017) Evaluation on the effects of wax inhibitor and optimization of operating parameters for wax deposition in Malaysian crude oil. Pet Sci Technol 35(20):1945-1950

Saeed MO, Azizli K, Isa MH, Bashir MJ (2015) Application of CCD in RSM to obtain optimize treatment of POME using Fenton oxidation process. J Water Process Eng 8:e7-e16

Saha SP, Mazumdar D (2019) Optimization of process parameter for alpha-amylase produced by Bacillus cereus amy 3 using one factor at a time (OFAT) and central composite rotatable (CCRD) design based response surface methodology (RSM). Biocatal Agric Biotechnol 19:101168

dos Santos DM, de Lacerda Bukzem A, Campana-Filho SP (2016) Response surface methodology applied to the study of the microwave-assisted synthesis of quaternized chitosan. Carbohydr polym 138:317-326

Song C, Kitamura Y, Li S (2014) Optimization of a novel cryogenic $\mathrm{CO} 2$ capture process by response surface methodology (RSM). J Taiwan Inst Chem Eng 45(4):1666-1676

Tanyildizi MS, Özer D, Elibol M (2005) Optimization of $\alpha$-amylase production by Bacillus sp. using response surface methodology. Process Biochem 40(7):2291-2296

Temoçin Z, Yiğitoğlu M (2009) Studies on the activity and stability of immobilized horseradish peroxidase on poly (ethylene terephthalate) grafted acrylamide fiber. Bioprocess Biosyst Eng 32(4):467-474

Torğut G, Tanyol M, Meşe Z (2020) Modeling and optimization of indigo carmine adsorption from aqueous solutions using a novel polymer adsorbent: RSM-CCD. Chem Eng Commun 207(8):1157-1170

Usman A, Aziz A, Abu-Noqta O (2019) Effect of independent variables on the stability of the synthesized gold nanoparticles using central composite design. Mater Today Proc 17:937-945

Wang Z et al (2020) Synthesis and water absorbing properties of KGMg-P (AA-AM-(DMAEA-EB)) via grafting polymerization method. Polym Sci Ser B 62(3):238-244

Wani TA, Ahmad A, Zargar S, Khalil NY, Darwish IA (2012) Use of response surface methodology for development of new microwellbased spectrophotometric method for determination of atrovastatin calcium in tablets. Chem Central J 6(1):1-9

Publisher's Note Springer Nature remains neutral with regard to jurisdictional claims in published maps and institutional affiliations. 